\title{
Rede cegonha: avanços e desafios da gestão no ambiente hospitalar
}

\author{
Stork network: advances and challenges of management in the hospital environment
}

\author{
Stork network: avances y desafíos de la gestión en el entorno hospitalario
}

\begin{abstract}
Marcondes Mateus Barbosa ${ }^{*}$, Elem Cristina Rodrigues Chaves ${ }^{2}$, Daniela Silva Leite ${ }^{3}$, Ana Helena Correa Quaresma ${ }^{3}$, Gabriela Paracampo de Albuquerque ${ }^{4}$, Ilka Lorena de Oliveira Farias Costa ${ }^{5}$, Ana Paula Oliva Reis ${ }^{5}$, Severino Azevedo de Oliveira Júnior ${ }^{6}$, Maria Helena Rodrigues de Mendonça ${ }^{2,3}$, Sérgio Beltrão de Andrade Lima ${ }^{3}$.
\end{abstract}

\section{RESUMO}

Objetivo: Conhecer os avanços e desafios obtidos com a implementação da Rede Cegonha no ambiente hospitalar. Métodos: Trata-se de um estudo de revisão integrativa das bases de dados Biblioteca Virtual em Saúde (BVS), Scientific Electronic Library Online (SciELO) e Literatura Latino-Americana e do Caribe em Ciências da Saúde (Lilacs), com temas referentes à Rede cegonha, a partir de 2010. Resultados: Foram obtidos 9 artigos, na SciELO (55\%), e BVS e Lilacs (22,2\% cada). O ano de 2013 correspondeu a 55\% publicações e, 2010, 2011, 2012 e 2015 (22,2\% cada), sendo $66,7 \%$ de caráter qualitativo, 22,2\% documental e 11,1\% revisão bibliográfica. Dos artigos, 33,3\% são do Rio de Janeiro e, Minas Gerais, São Paulo com 22,2\%, Espírito Santo e Pernambuco com 11,1\%. Embora a Rede Cegonha (RC) seja uma, estratégia que assegura às mulheres o direito ao planejamento reprodutivo e a atenção humanizada à gravidez, estudos revelam vulnerabilidade quanto à assistência ao pré-natal. Considerações finais: Na RC, o acolhimento com dignidade é o principal objetivo, assim, há necessidades de implantar protocolo de Manchester Obstétrico para o melhor fluxo de atendimento, além de capacitar profissionais, visando o desenvolvimento de suas atividades qualidade dentro do Hospital.

Palavras-chave: Rede cegonha, Parto humanizado, Assistência materno-infantil.

\begin{abstract}
Objective: To know the advances and challenges obtained with the implementation of the Stork Network in the hospital environment. Methods: This is an integrative review study of the Virtual Health Library (VHL), Scientific Electronic Library Online (SciELO) and Latin American and Caribbean Literature on Health Sciences (Lilacs) databases, with themes related to the Stork Network, from 2010. Results: Nine articles were obtained in SciELO (55\%), and VHL and Lilacs (22.2\% each). The year 2013 corresponded to 55\% publications and, 2010, 2011, 2012 and 2015 (22.2\% each), being $66.7 \%$ qualitative, $22.2 \%$ documentary and $11.1 \%$ bibliographic review. Of the articles, $33.3 \%$ are from Rio de Janeiro and, Minas Gerais, São Paulo with $22.2 \%$, Espirito Santos and Pernambuco with $11.1 \%$. Although the Stork Network (CR) is a strategy that guarantees women the right to reproductive planning and humanized attention to pregnancy, studies reveal vulnerability regarding prenatal care. Final considerations: In CR, welcoming with dignity is the main objective, so there are needs to implement the Obstetric Manchester protocol for the best flow of care, in addition to training professionals, aiming at the development of their quality activities within the Hospital.
\end{abstract}

Keywords: Stork network, Humanized childbirth, Assistance mother-child.

\section{RESUMEN}

Objetivo: Conocer los avances y retos obtenidos con la implementación de la Red Stork en el entorno hospitalario. Métodos: Se trata de un estudio de revisión integrador de las bases de datos de la Biblioteca Virtual de Salud (VHL), la Biblioteca Electrónica Científica en Línea (SciELO) y las bases de datos de Literatura Latinoamericana y del Caribe sobre Ciencias de la Salud (Lilacs), con temas relacionados con la Red Stork, de 2010. Resultados: Se obtuvieron nueve artículos en SciELO (55\%), y VHL y Lilacs (22,2\% cada uno). El año 2013 correspondió al $55 \%$ de publicaciones y, 2010, 2011, 2012 y 2015 (22,2\% cada uno), siendo 66,7\% cualitativa, 22,2\% documental y $11,1 \%$ de revisión bibliográfica. De los artículos, el 33,3\% son de Río de Janeiro y, Minas Gerais, Sao Paulo con 22,2\%, Espirito Santos y Pernambuco con $11,1 \%$. Aunque la Red Stork (CR) es una estrategia que garantiza a las mujeres el derecho a la planificación reproductiva y a la atención humanizada para el embarazo, los estudios revelan vulnerabilidad con respecto a la atención prenatal. Consideraciones finales: En CR, la acogida con dignidad es el objetivo principal, por lo que hay necesidades de implementar el protocolo obstétrico de Manchester para el mejor flujo de atención, además de la formación de profesionales, con el objetivo de desarrollar sus actividades de calidad dentro del Hospital.

Palabras clave: Rede cegonha, Parto humanizado, Asistencia madre-hijo. INTRODUÇÃO

1 Universidade Federal do Pará (UFPA), Belém - PA. `E-mail: mmarcondes_mateus@hotmail.com

2 Centro Universitário Fibra, Belém - PA.

${ }^{3}$ Centro Universitário Metropolitano da Amazônia (UNIFAMAZ), Belém - PA.

${ }^{4}$ Universidade do Estado do Pará (UEPA), Belém - PA.

${ }^{5}$ Santa Casa de Misericórdia do Pará, Belém - PA.

${ }^{6}$ Secretaria Municipal de Saúde de Parnamirim, Parnamirim - RN. 
Instituída em 2011, a Rede Cegonha surge como uma estratégia do Sistema Único de Saúde (SUS) fundamentada nos princípios da universidade, equidade e integralidade da atenção à saúde materna infantil, onde visa estruturar e assegurar o acesso, acolhimento e atenção humanizada e segura à gravidez, ao prénatal, parto e ao puerpério. Ademais, visa proporcionar um nascimento e desenvolvimento seguro e saudável às crianças (BRASIL, 2011; CUNHA MAO, 2017).

No Brasil, o programa ocorre sendo implantado de forma gradativa nos estados e municípios, os quais são responsáveis diretos pela elaboração dos planos e execução financeira, seguindo critérios epidemiológicos balizados por indicadores como: razão de mortalidade materna, taxa de mortalidade infantil e densidade populacional, além do monitoramento da rede temática (BRASIL, 2011; ASSIS TR, et al., 2019).

Os indicadores relacionados à saúde materno-infantis são ferramentas essenciais para avaliação da situação de saúde de cada região, além de servir de base para o planejamento de políticas públicas e programas de saúde (CUNHA MAO, 2017; ASSIS TR, et al., 2019).

Dessa forma, a mortalidade materna é definida como indicador de qualidade da atenção à saúde e a evolução da saúde materna, e a mortalidade infantil, até o primeiro ano de vida, refletem situações de vulnerabilidade, ineficácia da assistência à gravidez e/ou dificuldade de acesso aos serviços. Portanto, tal avaliação fundamenta o contexto de distribuição das redes de forma efetiva e de acordo com as necessidades. De fato, reduzir a mortalidade materna e infantil requer um desempenho contínuo, sistêmico e conjunto dos entes federativos da tripartite e profissional da saúde (ASSIS TR, et al., 2019; HENRIQUES TRP, 2019)

Segundo a Organização Mundial da Saúde (OMS), cerca de 830 mulheres morrem todos os dias por complicações na gravides ou no parto e, alusivo ao Brasil, a razão da mortalidade materna (RMM) é elevada, apresentando cerca de 64,5 óbitos a cada 100 mil nascidos vivos no ano de 2017 (OMS, 2018). Todavia, embora o país tenha registrado um decréscimo de 8,4\% na RMM em 2018, tal redução ainda é considerado um desafio para os serviços de saúde (OMS, 2018; BRASIL, 2020).

Entre as ações da Rede Cegonha, com objetivo de fornecer uma assistência obstétrica qualificada e condutas baseadas em evidências no processo de parto e nascimento, os Centros de Parto Normal (CPN) visam reduzir as intervenções desnecessárias, os números de transferências para partos operatórios, índices de traumas perineais e mortalidades, sendo fundamentada no acompanhamento das gestantes por enfermeiras obstetras que oferecem um ambiente adequado e atendimento humanizado, contrapondo-se a cenários onde se conservavam os espaços sem privacidade e coletivos (CUNHA MAO, 2017; LOPES GC, et al., 2019). No mais, a contribuição da CPN não se fundamenta apenas no sentido de estrutura física, mas sim no processo de acolhimento qualificado e diferenciado (LOPES GC, et al., 2019).

Tal acolhimento, no contexto da saúde, se traduz em um conjunto de ações que visam melhorar o atendimento e aumentar a especificidade diante de determinada situação. $O$ ato de acolher está intrinsecamente ligado à atitude de ouvir atentamente o indivíduo e reconhecê-lo no seu todo, valorizando os aspectos biopsicossociais (SILVAR TJ e OMANO VF, 2015; FIGUEIROA MN, et al., 2017).

Assim, o acolhimento é norteado por princípios da assistência à saúde: garantia do acesso aos serviços à população, capacidade profissional quanto a resolutividade aos problemas e agravos da população; reorganização do processo de trabalho visando a descentralização dos serviços e o estabelecimento de uma relação humanitária (BRASIL, 2010; FIGUEIROA MN, et al., 2017).

Nesse contexto, há mais de duas décadas discute-se o modelo de atenção ao parto e sua expansão no Brasil com intuito de atingir padrões aceitáveis. Desse modo, sendo essa uma estratégia recém-implantada no SUS e com estudos ainda incipientes, corrobora a relevância de pesquisas que visem conhecer e expor os avanços e contribuições da Rede Cegonha à sociedade, além fornecer subsídios a gestores, profissionais e pesquisadores da saúde de modo a contribuir para prática dessa política. O presente estudo tem por objetivo apresentar os achados em literatura sobre os avanços e desafios obtidos com a implementação da Rede Cegonha no ambiente hospitalar. 


\section{MÉTODOS}

Trata-se de um estudo de revisão integrativa, conduzida a partir das bases de dados indexadas à BVS, SciELO e Lilacs com temas referentes à Rede cegonha.

Como critério de inclusão, foram utilizados trabalhos publicados em periódicos ou em formato de artigos, monografia, dissertações e/ou teses na língua portuguesa, publicados a partir de 2010 a 2020, cuja busca ocorreu através dos descritores: Rede Cegonha, Saúde da Mulher e Acolhimento, de forma agrupada ou isolada. No que tange aos critérios de exclusão, foram considerados o distanciamento com o objetivo da pesquisa.

Após triagem, foram encontrados 44 artigos relacionados ao tema da Rede Cegonha, os quais seguiram para avaliação de conteúdo e, aplicando os critérios de inclusão e exclusão, foram selecionados 9 artigos cuja abordagem proporcionou fazer uma ligação com a pesquisa realizada

Após o levantamento bibliográfico, dividiram-se os artigos em categorias para realização da discussão, sendo agrupados de acordo com título, autoria, local, ano, descritor e abordagem. Para avaliação e formatação de tabelas, foi utilizado o utilizado a Microsoft Excel versão 2016 (Figura 1).

Figura 1 - Organograma da estratégia metodológica para busca da revisão integrativa em bases de dados.

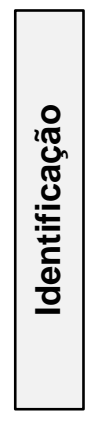

Trata-se de um estudo de revisão integrativa

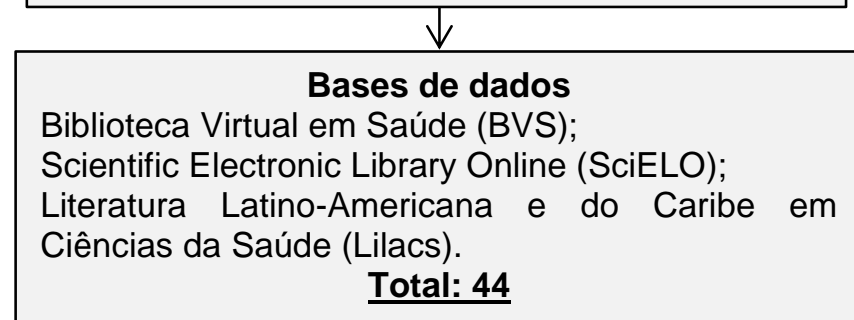

\begin{tabular}{|l|}
\hline 토 \\
엄 \\
즌 \\
\hline
\end{tabular}

Critério de inclusão: trabalhos em periódicos, artigos, monografia, dissertações e/ou teses na língua portuguesa, no período de 2010 a 2020;

Critérios de exclusão: distanciamento com o objetivo da pesquisa.
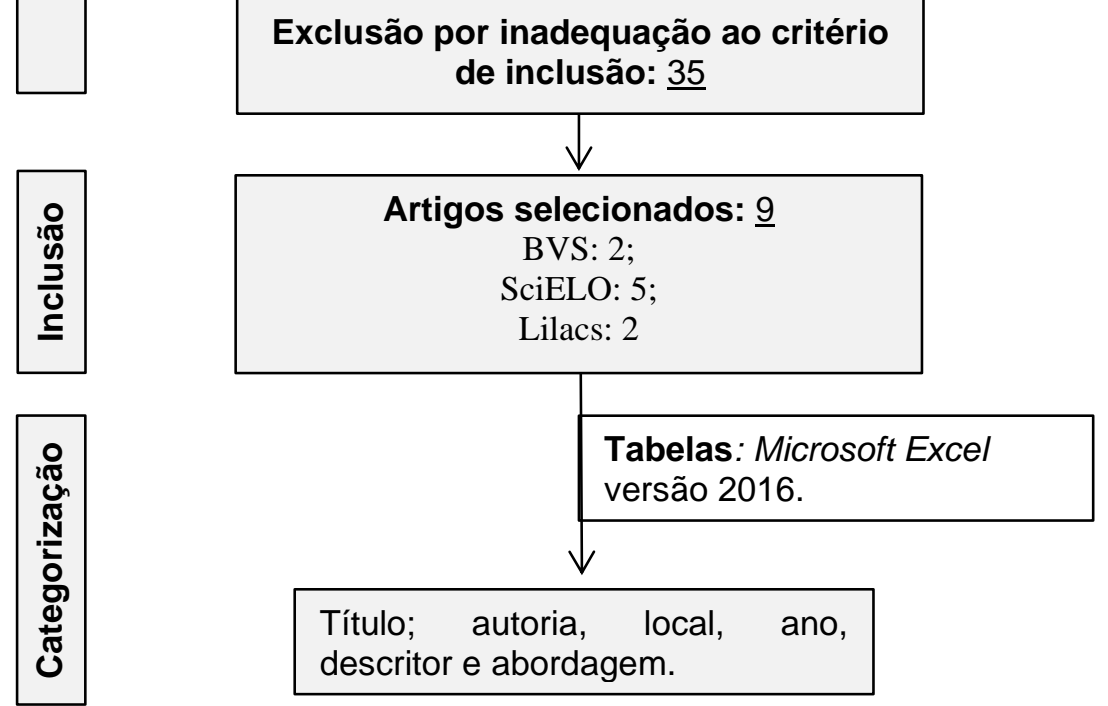

Título; autoria, local, ano,
descritor e abordagem.

Fonte: Barbosa MM, et al., 2021. 


\section{RESULTADOS E DISCUSSÃO}

Para uma melhor compreensão, os dados foram organizados e descritos com vista em algumas características estabelecidas (Quadro 1). De acordo com os dados, nas bases de dados consultadas, alguns artigos se sobrepuseram em duas bases ao mesmo tempo, apesar disso, foram obtidos nove (09) artigos, com maior quantitativo de estudos sobre Política de Saúde e Programa Academia da Saúde encontram-se no SciELO com 55\% dos trabalhos selecionados, BVS com 22,2\% e Lilacs, 22,2\%. Em relação aos descritores, 60\% foram buscados utilizando o termo 'Rede Cegonha', 20\% 'Acolhimento' e 20\% 'Saúde da Mulher'.

Referente ao ano de publicação, 2013 surge com destaque, sendo responsável por 55\% das publicações, seguido de 2010, 2011, 2012 e 2015, com 22,2\% cada. Observa-se que, embora a Rede Cegonha tenha sido lançada somente em 2011, o ano de 2010 já contava com políticas públicas voltadas à Saúde da Mulher e diretrizes organizacional da Rede de Atenção à Saúde no contexto SUS. A partir da análise temporal das publicações, foi possível observar a maior frequência de artigos de caráter qualitativa, com abordagem quali-quantitativa $(66,7 \%)$, seguido da pesquisa documental $(22,2 \%)$ e revisão bibliográfica $(11,1 \%)$.

Ao analisar o local de publicação dos artigos, foi observado que a maior parte das publicações referentes à temática advém da região Sudeste, sendo 33,3\% elaboradas no Rio de Janeiro, 22,2\% em Minas Gerais, 22,2\% São Paulo, 11,1\% em Espírito Santo e, na região Nordeste, com apenas 11,1\% de Pernambuco. Tais dados permitem, portanto, estabelecer a carência de estudos às demais regiões e, consequentemente, a necessidade de desenvolver novas pesquisas tendo em vista sua relevância no enfrentamento da mortalidade materna e violência obstétrica (HENRIQUES TRP, 2019). 
Quadro 1 - Relação dos estudos exportados das bases de dados segundo as características da pesquisa.

\begin{tabular}{|c|c|c|c|c|c|}
\hline$n^{\circ}$ & Título & Autor/Ano & Local & Descritor & Abordagem \\
\hline 1 & $\begin{array}{l}\text { Adequação da atenção à saúde da mulher e da } \\
\text { criança no município do Paudalho segundo o } \\
\text { olhar da rede Cegonha }\end{array}$ & $\begin{array}{l}\text { Alves MLP } \\
\qquad(2012)\end{array}$ & Pernambucco & Saúde da Mulher & Qualitativa \\
\hline 2 & $\begin{array}{l}\text { Adequação do processo de assistência pré-natal } \\
\text { segundo os critérios do Programa de } \\
\text { humanização do Pré-natal e Nascimento e Rede } \\
\text { Cegonha }\end{array}$ & $\begin{array}{l}\text { Martinelli KG, et al } \\
\qquad(2013)\end{array}$ & Espírito Santo & Rede Cegonha & $\begin{array}{l}\text { Pesquisa qualitativa e } \\
\text { quantitativa }\end{array}$ \\
\hline 3 & $\begin{array}{l}\text { Avaliação da Ferramenta Protocolo no 22: Ação } \\
\text { Rede Cegonha do Departamento Nacional de } \\
\text { Auditoria do Sistema Único de Saúde }\end{array}$ & $\begin{array}{l}\text { Costa JM } \\
(2015)\end{array}$ & Rio de Janeiro & Rede Cegonha & Pesquisa Documental \\
\hline 4 & $\begin{array}{l}\text { Dilemas antropológicos de uma agenda de saúde } \\
\text { pública: Programa Rede Cegonha, pessoalidade } \\
\text { e pluralidade }\end{array}$ & $\begin{array}{l}\text { Carneiro RG } \\
\qquad(2013)\end{array}$ & São Paulo & Rede Cegonha & Qualitativa \\
\hline 5 & Um modelo lógico da Rede Cegonha & $\begin{array}{l}\text { Cavalcanti PCS, et al } \\
\qquad(2013)\end{array}$ & Rio de Janeiro & Rede Cegonha & Pesquisa Documental \\
\hline 6 & $\begin{array}{l}\text { Estratégias de integração das práticas } \\
\text { assistenciais de saúde e de vigilância sanitária no } \\
\text { contexto de implementação da Rede Cegonha }\end{array}$ & Fernandes RZS (2013) & São Paulo & Rede Cegonha & Qualitativa \\
\hline 7 & $\begin{array}{l}\text { A humanização da assistência ao parto na } \\
\text { percepção de estudantes de medicina }\end{array}$ & $\begin{array}{l}\text { Rebello MTMP; Neto JFR } \\
\qquad(2010)\end{array}$ & Minas Gerais & Acolhimento & $\begin{array}{l}\text { Qualitativa, exploratório- } \\
\text { descritivo }\end{array}$ \\
\hline 8 & $\begin{array}{l}\text { Prática do acolhimento na assistência pré-natal: } \\
\text { limites, potencialidades e contribuições da } \\
\text { enfermagem }\end{array}$ & $\begin{array}{l}\text { Gonçalves ITJP } \\
\qquad(2013)\end{array}$ & Minas Gerais & Acolhimento & Revisão Narrativa \\
\hline 9 & $\begin{array}{l}\text { A coordenação da atenção ao pré-natal e ao } \\
\text { parto por equipes de saúde da família no } \\
\text { município do Rio de Janeiro }\end{array}$ & $\begin{array}{l}\text { Noel MM } \\
(2013)\end{array}$ & Rio de Janeiro & Saúde da Mulher & $\begin{array}{l}\text { Pesquisa quantitativa e } \\
\text { qualitativa }\end{array}$ \\
\hline
\end{tabular}

Fonte: Barbosa MM, et al., 2021. 
Após análise dos dados, pode-se caracterizar o material pesquisado em duas categorias: Avanços e Desafios da Rede Cegonha e Humanização da Assistência e Práticas de Acolhimento.

\section{Avanços e Desafios da Rede Cegonha}

A Rede Cegonha, instituída no SUS, estruturada pelo Ministério da Saúde, visa inserir uma rede de atenção integral fim de garantir o planeamento reprodutivo de forma humanizada e acolhedora às mulheres durante o processo de gravidez, parto e puerpério (após o parto), além de permitir e fornecer às crianças o nascimento e desenvolvimento de maneira segura e saudável (BRASIL, 2011; CUNHA MAO, 2017; FIGUEIROA MN, et al., 2017). Apesar dos avanços e reconhecimento da Rede Cegonha, estudos revelam a vulnerabilidade quanto à assistência ao pré-natal que variam desde o nível organizacional dos serviços no contexto de puérperas, com uso desnecessário de procedimento e ferramentas até questões de estruturas físicas inadequadas, dessa forma, compreende tal problemática como uma questão de saúde pública, de agenda política e governamental (ALVES AG, et al., 2017).

Segundo Alves MLP (2012), é preciso que os profissionais da Atenção Primária à Saúde executem suas ações de forma a promover a segurança e, sobretudo, adotar a responsabilidade pelos cuidados da gestante desde o início da gravidez até a alta pós-parto; por este motivo é essencial que ocorra uma parceria entre os serviços de atenção primária a saúde com os serviços responsáveis pela rede de atenção ao parto. $O$ autor ainda corrobora que as ações devem ser desenvolvidas assegurando 0 atendimento qualificado e humanizado, focando no indivíduo em sua totalidade e considerando fatores ambientais e socioeconômicos (ALVES MLP, 2012).

Nessa perspectiva, a literatura revela que, embora uma parcela dos profissionais da saúde execute suas ações baseando-se nos protocolos adotados nas unidades de saúde como garantia para o exercício seguro da função, ainda há certa resistência quanto à adesão aos protocolos, a falta de capacitação e percepção do trabalho em equipe, sendo esses pontos decisivos no quesito atenção gestacional de qualidade (RODRIGUES EM, et al., 2011; ALVES MLP, 2012; ALVES AG, et al., 2017). Estudo realizado por Carneiro RG (2013) expõem o país como recordista na incidência de cesárias por ano, onde se preconiza uma taxa de $5 \%$ a $15 \%$, diante disso, foram introduzidas medidas como Pacto Nacional pela Redução da Mortalidade Materna e Neonatal e a Política Nacional pelo Parto Natural e Contra as Cesáreas Desnecessárias, em 2008. Essa realidade traz à tona inúmeras críticas às práticas no âmbito obstétrico e afirma o mérito do parto humanizado.

Em uma visão ao acesso aos serviços, levando em consideração a elevada utilização do SUS pela população feminina, Alves MLP (2012) relata em seu estudo que o comprometimento da mulher surge a partir do momento em que há um acesso adequado ao serviço pré-natal e as propostas do programa são informadas, e como consequência, fornece um sentimento de integração e responsabilidade pelo cuidado gerando, de certa forma, o compartilhamento de responsabilidade entre gestantes e profissionais. Tais elementos são fundamentais e contribuem significativamente para humanização na atenção obstétrica. No entanto, essa realidade está longe de ser alcançado, visto que o atendimento a gestantes continua sendo executado de forma fragmentada, impessoal e sem diálogo pela maioria das equipes de saúde. Logo, a reversão desse quadro deve ocorrer com a sistematização do atendimento pré-natal, tendo a humanização como real direcionador do atendimento e a atenção sendo focalizada nas mulheres mais vulneráveis (ALVES MLP, 2012; MARTINELLI KG, et al., 2013).

Todavia, segundo Costa JM (2015), este olhar parcial traz um viés que dificulta uma real compreensão de como a política pública se encontra, dificultando a tomada de decisão pelo gestor. Nesse contexto, movimentos de saúde exigem o acesso à saúde às mulheres na medida em que questões de subjetividade torna-se uma miríade, a saber, um enfeixamento de inúmeros marcadores sociais a serem ponderados em suas particularidades. Assim, sobre o direito à saúde, é necessário ponderar a respeito das crenças, habitat e etnia, e não mais somente do corpo e dos aspectos emocionais, tematizar as diferenças culturais que, porventura, possam ser ali encontradas (CARNEIRO RG, 2013).

Destarte, outro ponto relevante refere-se às dificuldades da estratégia correspondente a falta de descrição das ferramentas e elementos, além da imprevisão das ações voltadas ao planejamento e 
vigilância familiar quanto aos óbitos de mulheres e crianças. Portanto, é essencial rever a teoria da Rede Cegonha visando detalhar e ampliar seu foco e ações, o que pode aumentar suas chances de sucesso (CAVALCANTI PCS, et al., 2013). Para Fernandes RZS (2013) as dificuldades na integração destas práticas podem estar relacionadas com desconhecimento dos gestores quanto ao "Papel da VISA" (Vigilância em Saúde), categoria que também apareceu na literatura.

Com base no exposto, é de grande relevância que a gestante e seu acompanhante recebam as orientações das ações e processos de trabalho para que ambos possam ter uma percepção de como funciona a Rede de atenção através dos pontos de atenção à saúde materna-infantil, de maneira que cada um saiba seus direitos e deveres (CAVALCANTI PCS, et al., 2013). Assim, esse processo irá facilitar e beneficiar a todos e ainda garantir a melhora da qualidade da assistência oferecida a gestante e a equipe. Podendo, de forma clara e objetiva, realizar os avanços desejáveis, partindo dos pontos críticos encontrados no cotidiano dos atendimentos da equipe, nas consultas, reuniões e até mesmo envolver a comunidade de forma sistematizada através da associação localizada. Melhorando ainda mais o acesso de forma eficaz à saúde (CAVALCANTI PCS, et al., 2013; FERNANDES RZS, 2013).

Contudo, cabe fortalecer junto aos gestores da saúde capacitações e fortalecimento do controle social para que seja realizado o seu papel in loco a fim de melhorar cada vez mais os serviços prestados. Buscando o comprometer em: prevenir, cuidar, proteger, tratar e produzir saúde. Ainda mais que, muitos serão os desafios a serem enfrentados quando lidando diretamente com a defesa da vida e com a garantia do direito ao acesso à saúde com dignidade e equidade (COSTA JM, 2015).

\section{Humanização da Assistência e Práticas de Acolhimento}

A humanização no qual se deve oferecer em serviços de saúde não se baseia em apenas fundamentar uma rede de diálogo entre profissional e a gestante, mas sim possibilitar a empatia e incentivar os meios de união e cooperação interdisciplinar de todos os envolvidos a fim de propor uma melhor convivência a nível hospitalar, e faz-se de forma que a qualidade desta assistência oferecida seja eficaz e eficiente no ambiente de trabalho, gestante e a família do paciente (REBELLO MTMP e NETO JFR, 2010; NOEL MM, 2013). Nesse sentido, corrobora pontos negativos anteriormente descritos como influenciadores ao tratamento desumano, impessoal e agressivo aos usuários dos serviços de saúde, sendo: incapacitação profissional, falta de condições técnicas e a infraestrutura inadequada, resultando em um serviço de baixa resolubilidade com tendência ao agravamento de uma situação já precária.

Estudos revelam que as experiências cotidianas com maior alvo de crítica nos serviços de saúde são as incapacidades dos profissionais na compreensão das demandas e as formas do atendimento, diante disso, sendo acolhimento público de qualidade um direito do cidadão e a necessidade de implementar uma cultura de humanização e reorganização da atenção ao parto, o MS, com a coparticipação de secretarias estaduais, municipais, gestores e profissionais de saúde, lançou o Programa Nacional de Humanização da Assistência Hospitalar (PNHAH) com propostas de ações integradas para fins de melhorias no processo de humanização dos serviços de saúde, tendo como objetivo principal aprimorar relações de profissionais entre si e para com usuários (BRASIL, 2001; REBELLO MTMP e NETO JFR, 2010).

No aspecto assistencial e de acolhimento ao trabalho de parto e nascimento, o movimento de humanização enfatiza o direito das mulheres ao parto seguro. Há décadas, a percepção de parto baseavase na medicalização, ou seja, tornava-o sob a concepção de doença ou problema médico no qual deveriam passar por monitoramento intensivo pela Medicina científica, sendo então encarregado ao profissional médico ofertar alguma forma de tratamento. Nesse modelo, autores destacam que as particularidades do nascimento tendem ao desrespeito e condutas inadequadas dos profissionais (REBELLO MTMP e NETO JFR, 2010; NOEL MM, 2013; CARVALHO SR, et al., 2015).

Gonçalves ITJP (2013), relata as noções de acolhimento como destaque devido sua contribuição à reorganização do SUS com vista na superação do modelo médico centrado nas verticalidades das ações, devido sua característica der triagem diferenciada e classificação de riscos a gestante, seguindo um olhar preciso e educativo que contribui para sua inserção em centros especializados. Sendo o nascimento um 
episódio de extrema importância para gestante e, devido ao impacto quanto às questões emocionais e físicas, torná-lo como uma experiência positiva é fundamental para estabelecer as concepções e vivências da maternidade (REBELLO MTMP e NETO JFR, 2010; GONÇALVES ITJP, 2011; NOEL MM, 2013).

No sentido à atenção pré-natal, sendo um processo essencial e marcado por ações de profiláticas, diagnóstica e curativa, foi realizado uma análise retrospectiva da cobertura de pré-natal no SUS. Durante o período de 2003 a 2009, houve avanços significativos no quantitativo de consultas oferecidas às gestantes, atingindo cerca de 19,4 milhões no ano de 2009, com aumento de 125\% em relação ao ano de 2003 quando foram registradas 8,6 milhões (GUERREIRO EM, et al., 2012). Em 2013, o Ministério da Saúde preconiza o mínimo de seis consultas de pré-natal com utilização de exames laboratoriais e de rotina, disponibilização de suplemento, tratamento e uso de vacinas, todos com registros na Caderneta da Gestante, garantindo uma assistência com detecção e assistência precoce (BRASIL, 2013).

Dados coletados do Sistema de Informações sobre Nascidos Vivos (Sinasc) demonstram que cerca de $66,5 \%$ das mulheres grávidas fazem sete ou mais consultas no pré-natal, tais dados corroboram a importância do SUS. Apesar do aumento de consultas, ainda é questionável a qualidade dessa assistência, haja vista a alta incidência de sífilis congênita em menores de um ano, com 26,3 mil casos confirmados em 2018, além do fato de a hipertensão arterial ser a causa mais frequente de morte materna no Brasil, os encaminhamentos inadequados ou tardios aos serviços de pré-natal de alto risco e o fato de a mortalidade materna brasileira ser ainda dez vezes maior que a de países desenvolvidos (BRASIL, 2019). Além disso, apenas $41,01 \%$ das gestantes inscritas no Programa de Humanização no Pré-Natal e Nascimento (PHPN) receberam a segunda dose ou a dose de reforço ou a dose imunizante da vacina antitetânica.

Destarte, as práticas diárias no ambiente de trabalho possibilitam obter uma melhor resolutividade dos objetivos quanto a um pré-natal seguro e de qualidade, um parto mais humanizado e tranquilo, oferecendo um conforto a essa equipe e a gestante em conjunto com seu acompanhante. No entanto, tal alteração se torna eficaz quando vigente um suporte técnico sob uma óptica assistencial sistemática a partir de protocolos e avaliações de ajustados forma participativa no modelo a produzir saúde (Gonçalves ITJP, 2013; Costa JM, 2015). Segundo Gonçalves ITJP (2013), o ato de acolher e humanizar são diretrizes fundamentadas na ética e políticas em produzir saúde, sendo uma ferramenta avançada à qualidade do SUS no sentido a eficácia e eficiência, além do seu impacto a formação de vínculos.

\section{CONSIDERAÇÕES FINAIS}

Na proposta da Rede Cegonha, o acolhimento e acesso com dignidade da gestante é o principal objetivo dessa estratégia, de modo a depender da participação ativa dos profissionais, gestores e usuários para reformulação dos serviços de saúde, recursos, organização e gerência para prestação de assistência. Dessa forma, programas e leis são criados com promessa de erradicar ou diminuir os impactos existentes na atenção à saúde, contudo, no ambiente Hospitalar, há necessidades de realizar a implantação do protocolo de Manchester Obstétrico para o melhor fluxo de atendimento e manejo adequado, além de capacitar profissionais para o bom desenvolvimento de suas atividades, visando a implantação do Núcleo de Educação, oferecendo qualidade dentro do Hospital.

\section{REFERÊNCIAS}

1. ALVES MLP. Adequação da atenção à saúde da mulher e da criança no município do Paudalho segundo o olhar da rede Cegonha. Recife, 2012:25.

2. ASSIS TR, et al. Implementação da Rede Cegonha em uma Regional de Saúde do estado de Goiás: o que os indicadores de saúde mostram sobre atenção materno-infantil? Reciis - Rev Eletron Comun Inf Inov Saúde, 2019; 13(4):843-53.

3. ALVES AG, et al. Política De Humanização Da Assistência Ao Parto Como Base À Implementação Rede Cegonha: Revisão Integrativa. Rev enferm UFPE on line., Recife, 2017; 11(2):691-702.

4. BRASIL. Ministério da Saúde. Secretaria de Assistência à Saúde Programa Nacional de Humanização da Assistência Hospitalar, 2001

5. BRASIL. Ministério da Saúde. Acolhimento nas práticas de produção de saúde. Núcleo Técnico da Política Nacional de Humanização, 2010; 2(4). 
6. BRASIL. Portaria no 1.459 , de 24 de junho de 2011. Institui, no âmbito do Sistema Único de Saúde - SUS - a Rede Cegonha, 2011.

7. BRASIL. Ministério da Saúde. Atenção ao pré-natal de baixo risco, 2013; 1(32): 318.

8. BRASIL. Ministério da Saúde. Protocolo Clínico e Diretrizes Terapêuticas para Prevenção da Transmissão Vertical do HIV, Sífilis e Hepatites ViraiS. Brasília, 2019.

9. BRASIL. Ministério da Saúde. Mortalidade Materna no Brasil - Boletim Epidemiológico n.ำ 20/MS, 2020.

10. CARNEIRO RG. Dilemas antropológicos de uma agenda de saúde pública: Programa Rede Cegonha, pessoalidade e pluralidade. Interface Botucatu, 2013; 17(44): 49-59.

11. CARVALHO SR, et al. Medicalização: 1251 uma crítica (im)pertinente? Physis Revista de Saúde Coletiva, Rio de Janeiro, 2015; 25(4): 1251-1269.

12. CAVALCANTI PCS, et al. Um modelo lógico da Rede Cegonha. Physis, 2013; 23(4):1297-1316.

13. CUNHA MAO. Análise da política Rede Cegonha: a atenção ao pré-natal e ao parto e nascimento no Recife, 2011 a 2015. Recife, 2017.

14. FERNANDES RZS. Estratégias de integração das práticas assistenciais de saúde e de vigilância sanitária no contexto de implementação da Rede Cegonha. Ciênc. saúde coletiva, 2014;19:11.

15. FIGUEIROA MN, et al. Acolhimento do usuário e classificação de risco em emergência obstétrica: avaliação da operacionalização em maternidade-escola. Escola Anna Nery 21(4) 2017.

16. GONÇALVES ITJP, et al. Prática do acolhimento na assistência pré-natal: limites, potencialidades e contribuições da enfermagem. Review Article, 2013;14(3).

17. GUERREIRO EM, et al. O cuidado pré-natal na atenção básica de saúde sob o olhar de gestantes e enfermeiros. Reme - Rev. Min. Enferm, 2012;16(3): 315-323.

18. HENRIQUES TRP. Determinantes dos óbitos infantis hospitalares e não hospitalares nos municípios do Vale do Jequitinhonha, Minas Gerais- Belo Horizonte, 2019.

19. LOPES GC, et al. Atenção ao parto e nascimento em hospital universitário: comparação de práticas desenvolvidas após Rede Cegonha. Rev. Latino-Am. Enfermagem, Ribeirão Preto, 2019;27:29.

20. MARTINELLI KG, et al. Adequação do processo da assistência pré-natal segundo os critérios do Programa de Humanização do Pré-natal e Nascimento e Rede Cegonha. Rev Bras Ginecol Obstet. 2014; 36(2):56-64.

21. NOEL MM. A coordenação da atenção ao pré-natal e ao parto por equipes de Saúde da Família no município do Rio de Janeiro. Dissertação (Mestre em Saúde Pública). Rio de Janeiro, 2013.

22. COSTA JM. Avaliação da Ferramenta Protocolo n.ำ 22 - Ação Rede Cegonha do Departamento Nacional de Auditoria do Sistema Único de Saúde. Dissertação (Mestre em Saúde Pública). Brasília, 2015.

23. ORGANIZAÇÃO MUNDIAL DA SAÚDE. Folha informativa - Mortalidade materna, 2018.

24. REBELLO MTM, NETO JFR João Felício. A humanização da assistência ao parto na percepção de estudantes de medicina. Rev. bras. educ. med., 2012, vol.36, n.2, pp.188-197.

25. RODRIGUES EM, et al. Protocolo na assistência pré-natal: ações, facilidades e dificuldades dos enfermeiros da Estratégia de Saúde da Família. Rev Esc Enferm USP, 2011; 45(5):1041-7.

26. SILVAR TJ, MANO VF. Sobre o acolhimento: discurso e prática em Unidades Básicas de Saúde do município do Rio de Janeiro. Saúde Debate | rio de Janeiro, v. 39, n. 105, p.363-374, ABR-JUN 2015 